\title{
Longitudinal Assessment of Personal and Professional Development Competencies in Medical Students
}

J Gen Intern Med 36(8):2506-8

DOI: $10.1007 / \mathrm{s} 11606-020-05969-\mathrm{x}$

(C) Society of General Internal Medicine 2020

\section{INTRODUCTION}

In 2013, the Association of American Medical Colleges identified personal and professional development (PPD) as one of eight competency domains for medical students. ${ }^{1}$ PPD encompasses a range of skills, including stress management, adaptability, work-life balance, trustworthiness, and self-confidence, which students are expected to develop throughout training. ${ }^{1}$ Despite efforts to formalize PPD training, students are primarily exposed through the hidden curriculum, with little research on how to teach or assess these PPD competencies. $^{2,3}$

\section{METHODS}

We conducted a prospective longitudinal cohort study at the Pritzker School of Medicine (PSOM) between 2014 and 2018. We aimed to (1) evaluate changes in students' self-assessed PPD skills over four years of medical school, and (2) identify target areas for PPD programming. Following a literature review, we developed an assessment of proficiency in key PPD competencies, which was emailed to students every six months from August 2014 through May 2018. 1, 4, 5 Eight surveys (S1-S8) were administered. The survey consisted of 15 items in both the Personal Skills (i.e., help-seeking behavior) and Professional Skills (i.e., leadership, teamwork) domains, five items assessing attitudes toward self-reflection, and two open-ended prompts on PPD goals. Likert-scale responses were dichotomized, with proficient defined as $\geq 4 / 5$. Proficiency scores were reported as percentage of items rated as proficient within the domain. Standard descriptive statistics and generalized linear mixed (GLM) models were used to analyze changes in domain proficiency scores over eight time points and to compare the preclinical and clinical phases. The

Previous Presentations This research has been presented as a poster at the Society of General Internal Medicine 2019 Annual Meeting in Washington, DC on May 11, 2019, AMA ChangeMedEd 2019 in Chicago, IL, on September 19, 2019, and AAMC Learn Serve Lead in Phoenix, AZ, on November 11.2019

Received May 3, 2020

Accepted June 5, 2020

Published online June 25, 2020
GLM models accounted for clustering effects and repeated measures within students over time. Analyses were performed using SAS 9.4.

\section{RESULTS}

Ninety-three (96\%) students completed at least one survey and were included in the analysis. Respondents were 53\% male, 56\% White, 9\% African-American, and 9\% Latino. Response rates ranged from $38 \%$ at six months into third-year clerkships (S6) to $91 \%$ at six months into first year (S2).

Proficiency in the Personal Skills domain was lowest at the start of second year (S3, 72\%), decreased significantly from the prior survey (S2 79\% vs S3 72\%, $p<0.01$ ). Personal Skills proficiency peaked at the end of fourth year (S8, 84\%) (Fig. 1). Proficiency in Professional Skills was lowest throughout first year (79\%; S1, S2) and highest in fourth year (92\%; S7, S8), significantly increasing in the first half of third year (S5 83\% vs $\mathrm{S} 689 \%, p<0.01)$.

When comparing aggregate preclinical (S1-S4, first and second year) and clinical (S5-S8, third and fourth year) phase responses, a significant increase was found in Professional Skills ( 80 to $86 \%, p<0.01$ ) proficiency, while no change was seen in the Personal Skills domain (76.3 to $76.0 \%$, $p>0.05$ ) (Fig. 2).

When examining themes in the free response goals, conflict resolution, communication, maladaptive perfectionism, physical health/exercise, spirituality, and leadership emerged as potential focus areas for future programming.

\section{DISCUSSION}

Overall, our data suggest that self-assessed PPD proficiency during medical school is lowest during transition points, specifically at the start of second and third year, but maintains an overall upward trajectory. Aggregate data for the preclinical and clinical years showed increased professional but not personal proficiency as students progress through training. Medical students' declining ability to maintain personal wellness as training intensifies during clinical years likely contributes to high burnout rates in residency and practice. ${ }^{6}$ Study limitations include response bias, single institution survey, and reliance on self-assessments.

Curricular transition points present unique opportunities to implement targeted programming to help improve health and 


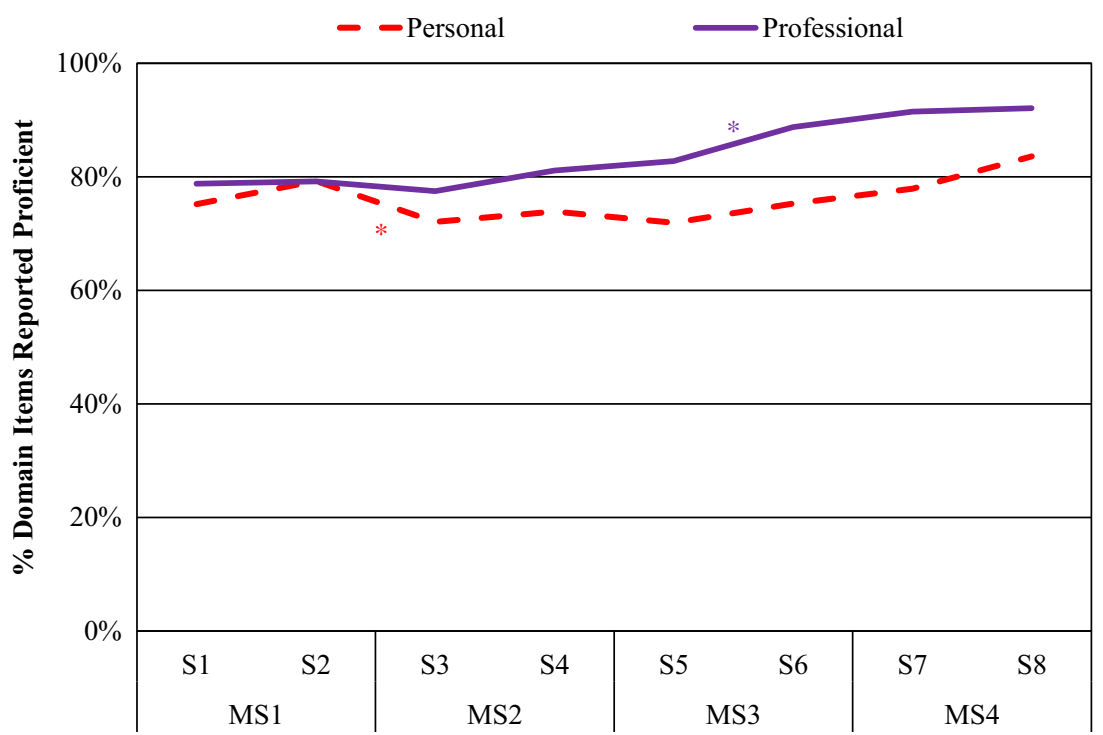

Figure 1 Trends in proficiency in the Personal Skills and Professional Skills domains over time for PSOM18. Percentage of competency items in each domain reported as "proficient" $(\geq 4 / 5)$ at each survey time point (S1-S8), with $* p<0.05$.

wellbeing, manage ambiguity, promote resilience, and address drivers of burnout in the learning environment. The optimal approach to professional development remains controversial.

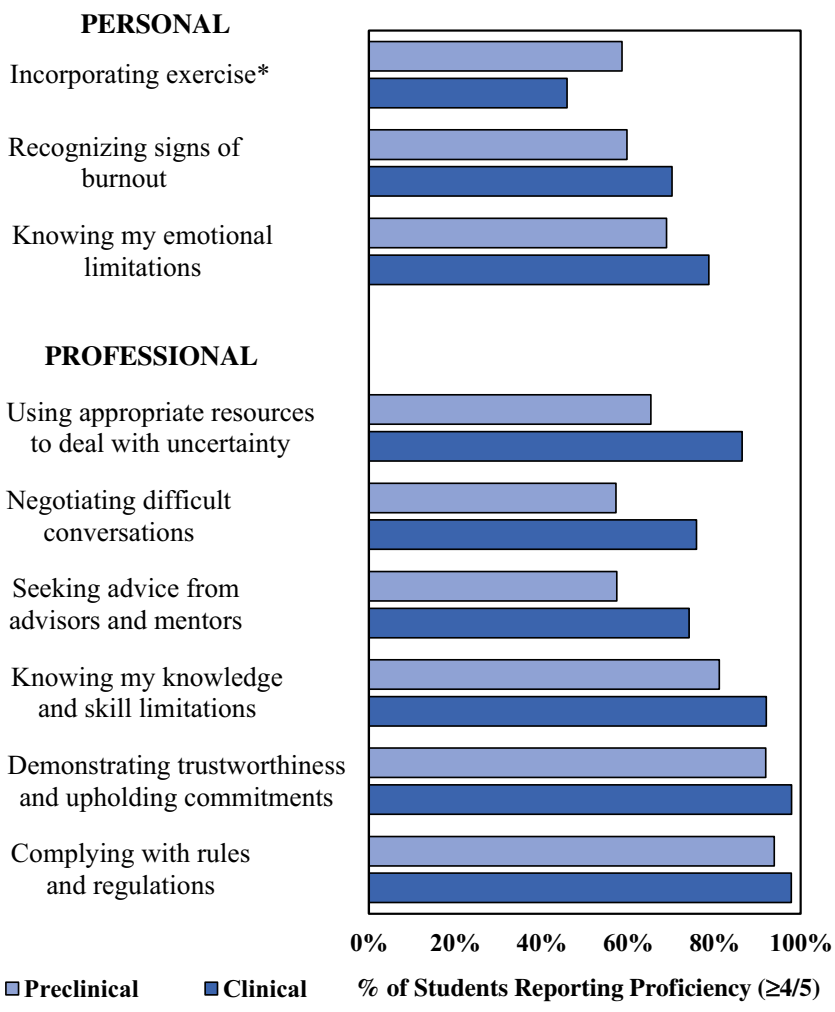

Figure 2 Competency items with significant changes in proficiency between the preclinical and clinical phases for PSOM18. Survey items within each domain with a significant difference in the percentage of students reporting proficiency $(\geq 4 / 5$ on Likert scale) between preclinical (S1-S4) and clinical (S5-S8) phases. Overall, 3/

15 skills in the Personal Skills domain, 6/15 skills in Professional Skills domain had a significant change. The asterisk indicates that all items listed statistically significant $(p<0.05)$, and all demonstrated increased proficiency over time except for "Incorporating exercise" which decreased.
The few studies that have examined approaches to assessing PPD suggest utilizing a multidimensional approach, encompassing evaluation from faculty, other members of the healthcare team, peers, and self-assessments from students. ${ }^{2,3}$ Our study provides a potential tool for schools to use to promote self-reflection, which can help guide coaching from advisors to promote the personal and professional development of students.

Future work will focus on developing targeted curricula to address the PPD competencies students identified as lower proficiency and initiatives to support students during critical transitions. Supporting personal and professional development as students navigate dynamic learning environments may be crucial in both fighting burnout in learners and training future clinicians prepared to tackle the complex problems of our health system.

Acknowledgments: The authors wish to thank Michael McGinty.

Hannah D. Caldwell, $B A^{1}$

Sandra A. Ham, $\mathrm{MS}^{2}$

Christopher D. Mattson, $M D^{3}$

James $N$. Woodruff, $M D^{4}$

Wei Wei Lee, MD, MPH

${ }^{1}$ University of Chicago Pritzker School of Medicine,

Chicago, IL, USA

${ }^{2}$ Center for Health and the Social Sciences, University of Chicago,

Chicago, IL, USA

${ }^{3}$ Department of Pediatrics, University of Chicago,

Chicago, IL, USA

${ }^{4}$ Department of Medicine, University of Chicago,

Chicago, IL, USA

Corresponding Author: Wei Wei Lee, MD, MPH; Department of Medicine, University of Chicago Chicago, IL, USA (e-mail: wlee6@uchicago.edu). 


\section{Compliance with Ethical Standards:}

Ethical Approval: The study was approved by the Institutional Review Board at the University of Chicago (IRB 160482).

\section{REFERENCES}

1. Englander R, Cameron T, Ballard AJ, Dodge J, Bull J, Aschenbrener CA. Toward a common taxonomy of competency domains for the health professions and competencies for physicians. Acad Med 2013;88(8): 10881094.

2. Drolet BC, Rodgers S. A comprehensive medical student wellness program-design and implementation at Vanderbilt School of Medicine. Acad Med 2010;85(1): 103-110.
3. Dyrbye LN, Sciolla AF, Dekhtyar M, et al. Medical School Strategies to Address Student Well-Being: A National Survey. Acad Med 2019;94(6):861868.

4. Frank J, Snell L, Sherbino J, eds. CanMEDS 2015 Physician Competency Framework. 2015. www.royalcollege.ca/rcsite/documents/canmeds/ canmeds-full-framework-e.pdf. Accessed 1 May 2020.

5. Simpson JG, Furnace J, Crosby J, et al. The Scottish doctor-learning outcomes for the medical undergraduate in Scotland: a foundation for competent and reflective practitioners. Med Teach 2002;24(2): 136-143.

6. Rotenstein LS, Ramos MA, Torre M, et al. Prevalence of Depression, Depressive Symptoms, and Suicidal Ideation Among Medical Students: A Systematic Review and Meta-Analysis. JAMA 2016;316(21):2214-2236.

Publisher's Note: Springer Nature remains neutral with regard to jurisdictional claims in published maps and institutional affiliations. 